\title{
Proceedings of the informal meeting on physics in agriculture
}

\author{
Wageningen, The Netherlands \\ 7-13 September, 1955
}

The increasing importance of physics for agriculture, and the similarity between the problems in agricultural physics studied in different parts of the world, formed an incentive for a number of physicists concerned with agriculture to discuss the present and the probable future role of physics in agriculture at an informal meeting held at Wageningen, The Netherlands, from the 7th to the 13th of September 1955. Evaporation in agriculture was the main subject for discussion.

The initiative in planning this meeting was taken by the following physicists :

Professor W. ReNtschler, Agricultural University, Stuttgart, Germany.

Dr. H. L. Penman, Rothamsted Experimental Station, Harpenden, Great Britain.

Dr. R. K. Schofield, Rothamsted Experimental Station, Harpenden, Great Britain.

Dr. D. A. dE Vries, Agricultural University, Wageningen, The Netherlands.

Professor W. R. van WIJא, Agricultural University, Wageningen, The Netherlands.

Professor BJ. BJERKE, Agricultural University, Ås, Norway.

Dr. E. M. LindBerg, Agricultural University, Uppsala, Sweden.

Professor Don Krrkuam, Iowa State College of Agriculture, Ames, Iowa, U.S.A. 
The Agricultural University of Wageningen gave moral and material support to the meeting. The interest shown by the Board of Governors of the University and in particular by the President Mr. J. M. van Bommel van Vloten, by the Secretary to the Board Dr. A. E. H. R. Boonstra, by the Rector Magnificus for 1955, Professor W. F. EijsvoogeL, and by several professors and other members of the staff was highly appreciated by those attending the meeting.

The Netherlands Ministry of Agriculture gave a generous subsidy and technical assistance. Mr. A. W. van der Plassche, Director General, Mr. A. H. JousTrA, Head of Foreign Relations at the Ministry, Messrs. A. H. HAAK and S. H. Tноо of the International Agricultural Study Centre, and Mrs. J. A. Frahm, Secretary to the Editorial Board of the Netherlands Journal of Agricultural Science, contributed much to the success of the meeting.

The Food and Agriculture Organization of the United Nations was informed about the project, and the Director of the Agriculture Division welcomed the initiative in arranging a contact between physicists active in agricultural science, and expressed his belief that such a meeting can be of great value to them.

Mr. M. DE NIET, Mayor of Wageningen welcomed the participants on a reception offered to them by the Municipal Authorities. 


\section{FINAL LIST OF PARTICIPANTS}

H. C. Aslyng,

Hydrotechnical Laboratory, Royal Veterinary and Agricultural College, 26, Rolighedsvej, Copenhagen, Denmark.

J. F. Bierhuizen,

Laboratorium voor Plantenphysiologisch Onderzoek, Rijksstraatweg 72, Wageningen, The Netherlands.

H. DE BOER,

Koninklijk Nederlands Meteorologisch Instituut, De Bilt, The Netherlands.

M. DE BoODT,

Rijkslandbouwhogeschool, Coupure Links 233, Gent, Belgium.

R Brouwer,

Botanisch Laboratorium, Grote Rozenstraat 31, Groningen, The Netherlands.

J. Businger,

Instituut voor Tuinbouwtechniek, S. L. Mansholtlaan 10, Wageningen, The Netherlands.

E. C. ChiLds,

School of Agriculture, University of Cambridge, Cambridge, England.

N. Collis-George,

School of Agriculture, University of Cambridge, Cambridge, England.

J. E. C. Coventry,

Rhodesia and Nyasaland Scientific Liaison Office, Africac House, Kingsway, London W.C. 2, England.

A. L. C. Davidson,

23 Collingham RD., London S.W. 5, England (temporary address).

L. J. L. DeIJ,

Koninklijk Nederlands Meteorologisch Instituut, De Bilt, The Netherlands.
J. F. Dugum,

Box 8100, Causeway, Salisbury,

S. Rhodesia.

L. F. Ennst,

Landbouwproefstation en Bodemkundig Instituut, Van Hallstraat 3, Groningen, The Netherlands.

M. Hallaire,

Institut National de la Recherche Agronomique, Route de Saint-Cyr, Versailles (Seine-et-Oise), France.

W. Hesse,

Institut für Agrarmeteorologie der Universität Leipzig, Arthur-Feistkorn-Strasze 3, Böhlitz-Ehrenburg b. Leipzig, Germany.

J. W. Holmes,

Division of Soils, C.S.I.R.O. Waite Institute, Adelaide, Australia.

G. Hygen,

Norges Landbrukshøgskole, Vollebekk, Norway.

† C. Kramer,

Koninklijk Nederlands Meteorologisch Instituut, De Bilt, The Netherlands. (Deceased Dec. 24, 1955).

C. Kvifte,

Fysisk Institutt, Norges Landbrukshøgskole, Vollebekk, Norway.

W. M. LEVI,

Meteorological Service for Israël, Lod-Airport, Israël.

J. M. Lyshede,

Det Danske Hedeselskabs Kulturtekniske Afdeling, Hydrometriske Undersøgelser, Skellet 1, Slagelse, Denmark.

A. MädE,

Zentral Institut für Angewandte Meteorologie, Grosze Steinstrasse 12, Halle/Saale, Germany. 
Mr. \& Mrs. M. Milosavljevic,

University of Belgrado, Dubljanska

94, Beograd, Yougoslavia.

\section{J. L. Monteith,}

Rothamsted Experimental Station, Harpenden, Herts, England.

P. Mortier,

Rijkslandbouwhogeschool, Gent, Belgium.

M. Olsen, Hedeselskabet, Viborg, Denmark.

P. K. Peerlkamp, Landbouwproefstation en Bodemkundig Instituut T.N.O., Van Hallstraat 3, Groningen, The Netherlands.

H. L. Penman,

Rothamsted Experimental Station, Harpenden, Herts, England.

R. W. Prunster,

Regional Pastoral Laboratory, Post Office Box 26, Deniliquin, S 6, N.S.W., Australia.

B. Ramsauer,

Bundes-Versuchsinstitut für Kulturtechnik und Technische Bodenkunde in Petzenkirchen, Österreich. Stubenring 1, Wien I, Austria.

W. Rentscirler, Institut für Physik und Meteorologie der Landw. Hochschule Hohenheim, Stuttgart-Hohenheim, Germany.

N. E. RIDER,

Meteorological Office, School of Agriculture, Cambridge, England.

R. K. Schofield,

Rothamsted Experimental Station, Harpenden, Herts, England.

K. Schubach,

Deutscher Wetterdienst, Bockenheimer Landstrasze 42, Frankfurt/M., Germany.
G. W. ScotT Blair,

National Institute for Research in Dairying, Shinfield, Reading, England.

I. Sestoft,

Det Danske Meteorologiske Institut, København K., Denmark.

R. O. Slatyer,

Land Research and Regional Survey Section, C.S.I.R.O., Box 109, City, Canberra, A.C.T., Australia.

L. P. SMith,

Meteorological Office M.O. 19, Headstone Drive, Harrow, England.

G. Stanhill,

National Vegetable Research Station, Wellesbourne, Warwick, England.

W. J. Staple, Soil Research Laboratory, Swift Current, Saskatchewan, Canada.

S. UHLIG,

Deutscher Wetterdienst, Kurhausstrasze 9, Bad-Kissingen, Germany.

H. J. Venema,

Laboratorium voor Plantensystematiek en -geografie, Landbouwhogeschool, Rijksstraatweg 37, Wageningen, The Netherlands.

D. A. DE VRIES,

Laboratorium voor Natuur- en Weerkunde, Landbouwhogeschool, Duivendaal 2, Wageningen, The Netherlands. ${ }^{*}$ )

G. P. WIND,

Centraal Instituut Landbouwkundig Onderzoek, Duivendaal, 10, Wageningen, The Netherlands.

-) Now : C.S.I.R.O. Division of Plant Industry, Regional Pastoral Laboratory, Deniliquin, N.S.W., Australia. 
F. WOELFLe,

Deutscher Wetterdienst, Bockenheimer Landstrasze 42, Frankfurt/M., Germany.

Mrs. R. I. WolfF,

Technion, Israël Institute of Technology, Haifa, Israël.
J. P. M. Woudenberg,

Koninklijk Nederlands Meteorologisch Instituut, De Bilt, The Netherlands.

W. R. van $W_{\mathrm{IJK}}$,

Laboratorium voor Natuur- en Weerkunde, Landbouwhogeschool, Duivendaal 2, Wageningen, The Netherlands.

\section{LADIES ACCOMPANYING PARTICIPANTS}

Mrs. Kvifte.

Mrs. Monteith.

Mrs. Mortier.

Mrs. Pennian.
Mrs. Scott Blair.

Mrs. Schofield.

Mrrs. Staple. 


\section{PROGRAMME OF THE MEETING}

Wednesday 7 th

14.00-14.15 Opening by Mr. J. M. v. Bommes van Vloten, President of the board of governors of the Agricultural University.

14.15-15.30 PENMAN - Evaporation, an introductory survey.

15.30-16.30 Discussions, discussion-leader : SCHOField.

20.00-22.00 Discussions, discussion-leader : SCHоғIELd.

Thursday 8 th

9.30-12.00 Short communications on evaporation :

Holmes, Monteith, Staple, Brouwer, Neale, Ramsauer, Hesse, Makkink, Slatyer, Businger.

Discussion-leader : SchOFIELD.

14.00-17.00 Short communications on miscellaneous subjects :

ScotT Blair, van Stralen.

Discussion-leader : Kvifte.

Mortier-de Boodt, Rentschlek.

Discussion-leader : Hesse.

Sestoft, Rentschler.

Discussion-leader : Mortier.

Boekel, Peerlkamp, Ernst.

Discussion-leader: CHInds.

20.00-22.00 Reception by the Mayor of Wageningen.

Friday 9 th Field trip to the North East Polder.

Saturday 10th

9.30-12.00 Short communications on miscellaneous subjects :

RENTSChLER.

Discussion-leader: ScotT BLAIR.

De VRIEs, Sterk.

Discussion-leader : Rentschler.

Free discussion on soil moisture :

Discussion-leader : Schofield.

14.00-17.00 Discussion on future cooperation of physicists in agriculture.

Introduction : vaN WIJk.

Discussion-leader : Schorield.

Discussion on education in physics and meteorology at the agricultural universities.

Introduction : RENTSCHLER.

Discussion-leader : SCHOFIELd.

Sunday 11th

14.00-18.00 Excursion to "De Hoge Veluwe" (National Park).

Monday 12th

$9.30-12.30$

Discussion on main subject.

Short communication by Halitaine.

Discussion-leader : SCHOFIELD.

Afternoon Field trip to the horticultural district of Aalsmeer.

19.30 Dinner for participants and guests.

Tuesday $13 t h$

9.30-12.00 Discussion on main subject, and conclusions arrived at the meeting.

Discussion-leader : ScHoField.

When the meeting was in progress a paper by Dr. L. Cavavillas in the Spanish language was received. It is incorporated in the proceedings of the meeting but it was not possible to have it discussed. 